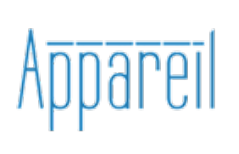

Appareil

3| 2009

Concerts publics et formes de la sensibilité musicale

\title{
Faire la musique
}

Enquête sur une création

\section{Denis Laborde}

\section{(2) OpenEdition \\ Journals}

Édition électronique

URL : http://journals.openedition.org/appareil/850

DOI : 10.4000/appareil.850

ISSN : 2101-0714

Éditeur

MSH Paris Nord

Référence électronique

Denis Laborde, "Faire la musique », Appareil [En ligne], 3 | 2009, mis en ligne le 08 juillet 2009, consulté le 30 juillet 2020. URL : http://journals.openedition.org/appareil/850 ; DOI : https://doi.org/10.4000/ appareil.850

Ce document a été généré automatiquement le 30 juillet 2020.

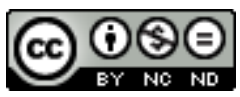

Appareil est mis à disposition selon les termes de la Licence Creative Commons Attribution - Pas d'Utilisation Commerciale - Pas de Modification 4.0 International. 


\title{
Faire la musique
}

\author{
Enquête sur une création
}

Denis Laborde

"It has been repeatedly intimated that there is a
difference between the art product, and the work
of art. The first is physical and potential; the
latter is active and experienced. It is what the
product does, its working [...] An esthetic
experience, the work of art in its actuality is
perception."
John Dewey, Art as experience

Dans un chalet en rondins de bois, quelque part dans la région de Rochester, dans le Vermont, un compositeur inscrit des notes, à l'encre noire, sur du papier à musique. Ce geste donne corps à un projet (la création d'une œuvre musicale) en donnant naissance à un programme : la partition. Ce geste ne sera pas sans conséquence. De ce qu'il aura permis de consigner dans les pages noircies à l'encre dépendront: le transit de dix tonnes de matériel d'une rive à l'autre de l'Atlantique, le montage (à Paris, Londres, Milan, Berlin ou Munich) de dossiers de financement public, la lutte fratricide de quelques festivals européens pour «la » création de l'œuvre, la survie de l'Ensemble Modern de Francfort, élu pour assurer cette création, l'opportunité, pour un jeune chef new-yorkais, de se faire connaître en Europe, un salaire pour les intérimaires du Hebbel Theater à Berlin, du Théâtre du Châtelet à Paris, ou du Muffathalle à Munich, des revenus garantis pour la société Licht de Nuremberg qui sera contactée pour la location de 120 projecteurs, le plaisir, pour les musiciens, de découvrir une partition inédite, de se mesurer avec l'intention d'un compositeur et de l'exécuter en public, l'occasion, pour ce public, d'activer une grille d'appréciation normative et de juger l'œuvre, le déclenchement de l'ire rageuse de critiques d'art qui traquent l'odieuse opération financière derrière le "commentaire sur l'ineffable » consigné dans le programme du concert, les angoisses d'un impresario londonien pressentant que l'œuvre ne serait pas achevée à temps, les inquiétudes d'une responsable du Festival d'Automne apprenant qu'effectivement l'inspiration n'avait pas guidé la main du compositeur avec la célérité promise, l'imagination débordante d'un directeur du festival Musica Viva, à Munich, 
pour compenser par un programme attractif l'opacité sémantique de l'œuvre musicale, le tout étant assuré par les avancées d'une technologie de pointe : les avions de British Airways qui conduisent le compositeur et son équipe par-delà l'Atlantique, les deux Volkswagen de l'Ensemble Modern qui transportent les instruments et quelques équipements scéniques depuis les sous-sols du 2, Schwedlerstraße, siège de l'ensemble, à Francfort, les trains de la SNCF qui amènent les musiciens à Paris, le métro qui, en fonctionnant normalement, permet aux spectateurs d'être au concert à l'heure dite, les techniques de fabrication d'images de synthèse, pour la bande vidéo, et d'échantillonnage aux sampler pour l'enregistrement électroacoustique, les performances d'un infaillible réseau téléphonique qui permettra de coordonner l'ensemble, les stratégies de marketing les mieux affûtées pour assurer la promotion de l'œuvre, et de son compositeur... Tout ça, parce qu'un jour, dans un chalet en rondins de bois, quelque part dans la région de Rochester, dans le Vermont, un compositeur aura inscrit des notes, à l'encre noire, sur du papier à musique. Sur quoi repose donc cette autorité que l'on prête au compositeur? Quel est ce type très particulier de croyance qui fait l'autorité de son geste créateur? Ces questions jalonnent mon propos. Je les formule, ici, à partir d'une implication personnelle dans ce projet, qui a pris la forme d'une observation participante, aux côtés des musiciens et de l'équipe technique de l'Ensemble Modern de Francfort.

2 Entre 1997 et 2002, j'ai pu enquêter, en effet, sur la création d'une œuvre musicale : un opéra-vidéo du compositeur américain Steve Reich (pour la musique) et de la vidéaste Beryl Korot (pour la vidéo projetée sur grand écran à l'arrière de la scène). L'œuvre s'appelle Three Tales (Trois Contes). Chaque conte forme l'argument d'un acte de cet opéra-vidéo. Premier acte : Hindenburg (l'incendie, en 1937, du zeppelin qui portait le nom du maréchal allemand, mis en rapport avec l'arrivée au pouvoir de Hitler, en 1933). Deuxième acte : Bikini (les essais nucléaires conduits dans l'atoll de Bikini avec ses conséquences cachées sur la population des îles). Troisième acte : Dolly (le clonage de la brebis écossaise et les questions éthiques qu'il pose). Un opéra engagé, qui dresse un bilan du $\mathrm{xx}^{\mathrm{e}}$ siècle sur le mode de la dénonciation. Des extraits du premier acte ont été donnés à entendre, en 1997 et en 1998. L'ensemble de l'opéra vidéo sera créé en 2001. Pour moi, c'est l'occasion de suivre, en direct, le processus de création d'une œuvre musicale, à la fois dans sa dimension artistique et dans sa dimension sociale ${ }^{1}$.

Comment toute une chaîne d'actions est-elle activée par ce geste créateur du compositeur? Comment passe-t-on du moment intime de la composition silencieuse à celui, public, du concert, ou plus exactement de la présentification de l'œuvre musicale? Je propose de m'arrêter ici sur trois étapes de la réalisation du projet : 1 . Le moment de la programmation; 2. Les répétitions ; 3 . Ernst Neisel, le régisseur. Une communication en forme de zoom, dans laquelle, à chacune des étapes, je change de focale ${ }^{2}$. Ce qui mérite deux précisions.

4 D'abord, il s'agit bien de trois séquences prélevées dans le processus de création musicale. Toutefois, ces trois séquences ne sont pas destinées, ici, à penser l'ordre logique d'une consécution d'existence qui mènerait $\mathrm{du}$ projet du compositeur à l'interprétation de l'œuvre au concert. De ce point de vue, bien d'autres situations pourraient être étudiées, quelques-unes le seront effectivement. Cet article est à lire comme l'état provisoire d'une enquête en cours. Je ne m'attarde donc pas à travailler un hypothétique lien de causalité qui relierait entre elles ces étapes de la création. Il s'agit simplement de quelques hapax dans le processus de création d'une œuvre. 
Ensuite, cette réduction d'échelle n'est pas synonyme d'une réduction de la complexité des situations étudiées. Le passage d'un point de vue macroscopique à un point de vue portant attention aux modalités de la présence dans l'espace des interactions dans lequel Ernst Neisel, le régisseur, se trouve impliqué, suppose un changement du type d'attention porté aux objets et aux relations. Ce changement de regard nous fait apercevoir d'autres types de complexité à l'œuvre à cette échelle "micro ». Réduire l'échelle d'observation ne revient donc pas à simplifier la tâche d'observation, elle la modifie ${ }^{3}$.

Autrement dit, je présente ici quelques moteurs de réflexion d'une recherche en cours, qui ne prendra fin qu'en 2001, avec la création de l'œuvre, à Vienne. Il s'agit, ici, de faire jouer des situations d'observation, des descriptions et des échelles dans un dispositif d'analyse qui n'est pas encore stabilisé et, en particulier, d'éprouver la rationalité du projet de Steve Reich et Beryl Korot en la mesurant, d'une part, à l'aune des pratiques qu'elle entend gouverner, et, d'autre part, à l'inventivité présente dans la réalisation des tâches prescrites. L'enjeu de cette recherche consiste dans une étude des dispositifs de représentation de l'espace d'action et de l'engagement du corps dans la situation, ce qui revient à accorder une place centrale à la perception. Le projet du compositeur en vient-il à se diluer dans les mille gestes qui réalisent son œuvre ? Ce travail s'efforce de quitter l'ordre de l'ineffable pour porter attention à ce que j'appellerai le second concert, celui qui se joue dans l'agencement des dispositifs technologiques et de l'action des agents impliqués dans la réalisation de l'œuvre.

\section{Steve Reich}

6 Au commencement, il y a le projet de Steve Reich, ou plutôt, il y a « Steve Reich » avec guillemets, c'est-à-dire ce "Steve Reich» que nous connaissons en raison de la familiarité que nous avons acquise avec ses œuvres. C'est le "Steve Reich » que nous lègue notre institution musicale. Au commencement, il y a donc «Steve Reich» et son projet : Three Tales ${ }^{4}$.

7 Né à New York, en 1936, Steve Reich est un homme à part. Il le revendique volontiers, en s'instituant en autoréférence. Sa particularité vient de ce qu'il a reçu une formation classique de compositeur à la prestigieuse Julliard School de New York, pour s'en écarter, ensuite, en allant étudier la percussion en Guinée, pendant deux ans. Cet itinéraire peu commun l'a incité à composer quelques hybrides qui sonnèrent d'une façon bien étrange dans les années soixante-dix. Steve Reich fut, avec Philipp Glass, à l'origine d'un courant qui irrigua le monde de la musique et Music for Eighteen Musicians fut, en 1974, l'œuvre par laquelle le syntagme « musique répétitive » émergea, avant de se voir validée, à la fois, par l'institution musicale et par les publics, et notamment le public qui, à cette époque, écoutait les Pink Floyd.

8 Cette "œuvre phare du minimalisme sophistiqué " (Pierre Gervasoni, Le Monde, 6 octobre 1997) fit toute la réputation de Steve Reich. Aujourd'hui, cette réputation précède le compositeur. Gageons qu'elle ne fut pas pour rien dans l'attention que les festivals européens témoignèrent à l'égard de son projet. Et, de fait, Music for Eighteen Musicians joue un rôle clé dans le dispositif de programmation de Three Tales. Je distingue deux étapes, et commence par la seconde.

9 L'opéra sera créé, à Vienne, le 11 mai 2001. Il sera ensuite repris à Londres (Barbican Center) le 10 septembre, à Paris (Festival d'Automne) le 13 septembre, à Turin 
(Settembre Musica) le 23 septembre, à Berlin (Hebbel Theater) le 29 septembre, à Lisbonne (Centro Cultural Belem) le 5 et le 6 octobre. Cette programmation a fait l'objet de négociations qui ont associé Steve Reich, Beryl Korot, Andrew Rosner (l'impresario londonien), Thomas Fichter, représentant de l'Ensemble Modern, de Francfort, et les directeurs des principaux festivals européens. La réunion s'est tenue le 19 septembre 1998, à 18 heures, dans une salle du Muffathalle, à Munich, dans le plus grand secret. Je n'ai pas eu le droit d'y assister : “It's business!", m'a dit Steve Reich, qui a clôturé ainsi l'espace de la négociation.

10 Cependant, toutes les personnes qui participaient à cette réunion se connaissaient déjà. $\mathrm{Au}$ moment même d'ouvrir cette réunion, les agents n'étaient plus les simples représentants anonymes d'institutions éparses. Ils étaient déjà des partenaires œuvrant à la réalisation d'un projet commun. Dès lors, la réunion de Munich ne vise pas une évaluation esthétique du projet. On n'argumente pas sur l'intérêt de Three Tales, sur la quantité d'innovation dont l'opéra est porteur... La discussion porte sur les conditions de réalisation. Non pas sur le projet, mais sur le programme : comment pouvons-nous faire pour créer du «Steve Reich »? Telle est la question.

11 C'est que cette seconde étape du projet - qui couvre la période 1998-2001 - a été précédée d'une première - 1996-1998 -, au cours de laquelle Steve Reich a composé Hindenburg, le premier acte. En 1997, dix minutes de ce premier acte ont été données à entendre dans plusieurs festivals d'Europe (Bonn, Londres, Amsterdam, Paris, Berlin). Le 19 septembre 1998, Hindenburg est donné à entendre, dans son ensemble (soit trente minutes), dans le cadre du festival Musica Viva, à Munich. Et voilà l'ingénieux marketing. Dans le programme des concerts de cette première période, Hindenburg était couplé avec Music for Eighteen Musicians, le tube. La création de Three Tales se fait donc par tuilage. Avant de gagner son autonomie, l'opéra vidéo existe en relation avec l'œuvre qui fit toute la réputation de Steve Reich, celle qui fabriqua «Steve Reich ». En 1997, et en 1998, c'est pour Music for Eighteen Musicians que le public vient au concert, et il vient nombreux. En 2001, c'est pour Three Tales qu'il viendra, et il viendra nombreux. C'est, en tout cas, le pari que font les organisateurs. Ainsi, c'est par Music for Eighteen Musicians que Three Tales entre progressivement dans notre univers culturel. L'innovation s'appuie sur le déjà connu.

12 D'autres instances, mobilisées par le projet, viennent stimuler l'option des organisateurs. "Steve Reich» et son projet musical n'attisent pas seuls l'intérêt des responsables de festival. Il y a aussi le propos. Beryl Korot veut faire de l'art vidéo un moyen de « réveiller la conscience humaine ». Steve Reich trouve qu'elle a raison, mais, en tant que compositeur, son projet est de faire œuvre musicale, pas "d'agiter le drapeau rouge ». Il travaille sur Three Tales comme il travaillerait sur n'importe quel autre projet. Il est simplement heureux de mettre sa science au service d'une œuvre commune avec Beryl Korot. Évidemment, le propos des Three Tales ne lui est pas indifférent. Il adhère totalement à l'indignation exprimée par Beryl Korot. Il s'investit dans ce projet-là, parce que, si Three Tales promet d'être un opéra engagé, il est au service de la bonne cause. Et dès lors qu'il est question de drames aussi considérables que ceux évoqués par Hindenburg, par exemple, comment ne pas être d'accord avec eux? Comment les inquiétudes qu'ils entendent exprimer, les questions qu'ils entendent porter dans l'espace public pourraient-elles ne pas être partagées, notamment, par les responsables de programmation des festivals? Gageons que cette dimension, à la fois éthique et civique, du projet n'entre pas pour rien dans 
l'engagement des institutions. Cette fois, le crédit ne vient pas d'un monde musical clos, refermé sur lui-même, mais de l'actualisation référentielle d'un principe éthique : la dénonciation du régime nazi. Le crédit apporté au projet vient d'une culture commune de «l'indignation éclairée », qui pose la connivence d'un partenariat.

13 Troisième instance dont on repère la mobilisation dans l'adoption du projet : la forme opéra vidéo est nouvelle, mais elle n'est pas inédite. Trois ans plus tôt, Beryl Korot et Steve Reich avaient composé un opéra vidéo sur Dachau : The Cave. Cette fois, il s'agit, en somme, d'élargir le propos déjà abordé dans The Cave. Il existe donc un précédent. En programmant Three Tales, les organisateurs ne font pas un saut dans l'inconnu. Ils savent qu'ils mobiliseront des réseaux de collaboration qui ont déjà fait leurs preuves.

On pourrait trouver bien d'autres instances mobilisées dans le dossier de Three Tales. Pour le moment, je me contente de ces trois, qui façonnent une spécificité de l'objet et conditionnent sa réalisation. Les axes - musico-médiatique, éthique, technique - sont les trois axes qui permettent aux organisateurs d'instaurer un lien avec "Steve Reich » en spécifiant les cadres d'une anticipation calculée, c'est-à-dire d'une mise en œuvre du projet.

Résumons : au commencement, il y a "Steve Reich", avec guillemets (c'est-à-dire le compositeur précédé de sa réputation) et le projet: un texte, des noms d'interprètes, des réseaux de diffusion qui ont fait leurs preuves, des lieux, des partenaires institutionnels et privés, des schémas, des plans, des photos, une vidéo (The Cave), un calendrier, une stratégie de diffusion, un budget, des réalisations antérieures ; en bref : une cartographie du projet. À cela vient s'ajouter l'annonce, par Sony, de l'édition en dix CD de l'intégrale de l'œuvre de Steve Reich pour l'année 1997. Comment ne pas y voir un partenariat possible ou, pour le moins, l'opportunité de voir se former une attente spécifique dans un public potentiel?

16 L'adhésion des festivals au projet de Steve Reich se fait sur la promesse de l'activation d'une chaîne de coopération qui fonctionnera avec toutes les chances d'aboutir: réputation de Steve Reich, implication de partenaires fiables avec lesquels des habitudes de travail sont déjà contractées (Ensemble Modern), professionnalisme des membres de la fondation Steve Reich de New York, faisabilité en termes d'innovation. Steve Reich garantit, en effet, que son œuvre se pliera aux conditions de réalisation (espace, temps, coûts, compétences) des théâtres d'accueil. Il faut y ajouter la mise en réseau de plusieurs festivals européens, qui permet de diminuer les coûts de production et de diffusion, et qui implique une compétition entre les festivals... en même temps qu'une uniformisation de leurs programmes. À la sortie de la réunion secrète de Munich, Neile Hartling, la directrice du Hebbel Theater de Berlin était très fière de m'annoncer : «J'ai gagné : nous aurons Steve Reich avant Paris ». Josephine Marcovits, directrice du Festival d'Automne, souffrante, n'avait pas pu assister à la réunion ${ }^{5}$.

Ainsi l'œuvre façonne-t-elle l'identité du festival au prix d'une double mise en série. À l'intérieur de chaque festival, d'abord, chaque création programmée est mise en réseau avec toutes celles qui sont inscrites au programme du festival. Chaque festival est ainsi singulier du point de vue de la programmation, de l'infrastructure et des habitudes du lieu. L'identité se conjugue ici sous la forme de la singularité. Mais la mise en réseau se fait aussi entre festivals, pour un allègement des coûts. De ce point de vue, il entre en relation avec tous les autres. L'identité se conjugue, cette fois, sous la forme de l'identique. 
18 Après avoir pointé quelques-unes des instances activées dans l'adhésion au projet du compositeur, examinons la réalisation. Pour que la promesse du projet se réalise, il faut un programme d'actions, c'est-à-dire une autre partition, qui n'est plus celle du compositeur mais celle de l'organisateur ${ }^{6}$. La partition de Steve Reich induit des comportements visant à faire exister l'œuvre d'art. La partition de l'organisateur du concert est de même nature: faire faire pour faire être. Elle formalise un plan qui s'organise en paramétrant le budget, les priorités qui ont été dégagées, les agents nécessaires, les possibilités techniques, les contraintes temporelles. Il postule l'existence d'autant de «boîtes noires» qui sont des équipes fonctionnant indépendamment les unes des autres et qui seront simplement mises en harmonie la veille du concert, sous l'autorité du compositeur (en fait de Steve Erhenberg, j'y reviendrai). Je propose, ici, d'ouvrir quelques-unes de ces « boîtes noires ».

\section{Fabriquer une création}

Le processus de création musicale assigne ainsi des tâches, définit des rôles, dessine des "catégories d'acteurs». La création d'une œuvre s'apparente au déclenchement d'un processus en chaîne qui recèle des conditions de félicité : une création réussie est une mobilisation réussie (Menger), une déviance légitimée (Becker). C'est, dans tous les cas, un processus institutionnel. Comme toute invention, elle n'est «ni de l'ordre de l'organigramme, ni seulement de l'ordre de la cognition : elle est celle d'une rationalité qui ne va pas sans une action distribuée » (Cohen, 1994, p. 90).

20 Cette action distribuée ne se fait cependant pas selon un ordre immuable, préréglé qui fonctionnerait automatiquement, conformément aux consignes émises par un hypothétique bureau des méthodes. La «mise en forme» de l'œuvre musicale et sa présentation au public (ce que l'on a coutume de désigner par le terme d'exécution et que je qualifierais, partageant en cela l'optimisme suggéré par Jacques Cheyronnaud, 1997, de réalisation vive) se fait au prix de multiples négociations et d'innombrables ajustements. Ces négociations et ces ajustements (ces médiations, pour reprendre le terme clé de la sociologie de la musique d'Antoine Hennion) ont leur espace privilégié : c'est l'espace de la répétition. J'étais à Munich pour la création du premier acte.

21 Le programme des répétitions avait été établi un an à l'avance par Monika Cordero, secrétaire de l'Ensemble Modern. Il se présente comme une synthèse des impératifs et des contingences. Le concert avait lieu à Munich le samedi 19 septembre 1998, les répétitions commencèrent le mardi 15 septembre, à Francfort. Mais déjà, le 14, Ernst Neisel, le régisseur de l'Ensemble, avait tout installé. Les répétitions de Hindenburg commencent le mercredi 16, avec la venue des chanteurs par le vol de $14 \mathrm{~h} 05$ en provenance de Londres.

La répétition est à 16 heures. Il est prévu qu'elle dure jusqu'à $21 \mathrm{~h} 30$. Une équipe de télévision filme : il y aura une annonce sur les chaînes nationales. À 20 heures, Steve Reich en a assez. Il est fatigué, dans le décalage horaire. Il est satisfait du résultat, propose qu'on en reste là, la décision fait l'unanimité.

Jeudi. On répète Hindenburg de 14 à 17 heures, mais Steve Reich trouve que les musiciens sont décidément très forts. À 16 heures, il propose qu'on en reste là, la décision fait l'unanimité. Il prend le train pour Bruxelles où l'une de ses œuvres est jouée le soir même. Steve Reich s'en va, les musiciens aussi : nous nous donnons 
rendez-vous le lendemain à la gare de Francfort pour le train de 11h09, destination : Munich.

Le compositeur est parti, les musiciens aussi, mais Monika Cordero est encore là et une intense activité règne dans les sous-sols de l'immeuble. Il s'agit de charger les deux camions de 7,5 tonnes qui partent avec le matériel d'orchestre. Pour le matériel scénique, Steve Ehrenberg apporte tout de New York. Quant aux 120 projecteurs loués à la société Licht, un camion est allé les chercher à Nuremberg. Dans les sous-sols, je rencontre : quatre chauffeurs, deux chefs des opérations scéniques, un ingénieur du son assistant. Ernst Neisel orchestre le déroulement des opérations en veillant à ce que rien ne manque. Il est, à ce moment, le trait d'union entre la plume du compositeur, l'interprétation du musicien et l'attente de l'auditeur. Il a des listes sans fin, des supports mnémoniques plein les poches, mais il refuse de me les montrer: "c'est privé ». Ici, dans les sous-sols, du côté de la technique, on est entré dans une autre temporalité. C'est le temps de la nuit qui tombe sur Francfort et d'une tension un peu mystérieuse : tension de la route à faire, tension des installations à faire à l'arrivée sur place, tension de la découverte d'un lieu qu'on ne connaît pas et de l'ajustement d'une tâche avec un plan d'action. Le temps, ici aussi, est minuté. Ernst Neisel tient le registre: "On s'en va à 20 heures, on commence à installer cette nuit, à 3 heures. Ensuite, c'est sans arrêt jusque dans la nuit de vendredi à samedi, sans doute vers 2 heures du matin ».

Vendredi. À 19 heures, tout le monde est dans le Muffathalle, à Munich. L'installation n'est pas terminée, mais les projecteurs sont en place, les instruments sont sur scène, les appareils de projection sont installés et les réglages sont faits. Steve Reich revient de Bruxelles, Beryl Korot est arrivée de New York, Steve Ehrenberg, l'éclairagiste aussi, et Jack Young est arrivé de Philadelphie. Tout le monde est là, réuni pour la première fois, et nous sommes à la veille du concert. Chacun s'installe pour durer dans la nuit. Peu à peu les repères s'effacent. Dans le Muffathalle, nous sommes coupés du monde extérieur. La suspension du temps présuppose un isolement topologique. Le temps s'étire, il paraît long, les musiciens parlent à mi-voix, l'espace de la scène se transforme en espace éclaté de négociations éparses. Les transactions ont lieu sur des modes divers, le silence gagne la salle de concert. Nous sommes une cinquantaine dans la salle, il fait froid, nous sommes mal installés au milieu des bouteilles vides de Coca-Cola, des mégots écrasés et des empilements de chaises. Tout est en place, il est 23 heures, c'est la première mise en présence de l'œuvre, la première "présentification ». Nick Mangano, le metteur en scène, donne ses dernières instructions, puis il s'efface. C'est Steve Erhenberg, l'éclairagiste, qui devient le seul maître des lieux : il donne le signal du départ à Jack Young, le projectionniste de Philadelphie, et nous assistons alors à la première "présentification» de l'œuvre. Nous sommes, pour la première fois, en présence de Hindenburg.

Jusqu'au lendemain, jusqu'au moment du concert, chacun va ajuster son action au projet collectif. Dans la prolifération des situations observables dans la salle de concert, on aperçoit une multiplicité d'emplois du temps, une multiplicité de comportements qui, peu à peu, convergent. Ces temporalités distinctes entrent en correspondance. Des faisceaux d'actions s'harmonisent à mesure que se réduisent les pôles de décision et qu'approche l'heure du concert. Norbert Ommer, l'ingénieur du son, est au point. Il a effectué tous les réglages, donné les dernières instructions aux musiciens, il se tait. Steve Reich et Beryl Korot sont à ses côtés autour de la console. Ernst Neisel, a tout 
rangé sur scène, il se déplace calmement désormais, attentif à la façon dont les musiciens, qui prennent place, s'adaptent au dispositif scénique. Bradley Lubman, le chef d'orchestre, est au pupitre, baguette en main, prêt à démarrer à tout moment. Nick Mangano, le metteur en scène, a réglé le salut final, puis il s'est assis, silencieux. Steve Erhenberg commande désormais, et lui seul. C'est lui qui évalue la situation et donne l'ordre à Jack Young, le projectionniste, de lancer la bande vidéo qui commande l'ensemble.

Au moment du concert, il faudra, à son tour, que Steve Erhenberg s'efface. Seul Bradley Lubman commande. C'est «le chef» d'orchestre. Dans le concert, le dispositif technologique doit s'effacer pour que chacun, dans le public, puisse cultiver l'apparence d'une immanence de l'œuvre d'art. L'univers de la croyance ne peut s'éployer qu'à la condition que s'efface le dispositif technique ${ }^{7}$. Alors, nous ne serons plus dans le temps privé des portes closes et de la répétition, mais dans celui, public, du concert. Le professionnalisme, c'est-à-dire la maîtrise des rouages et des procédures institutionnelles, aura permis que le concert ait lieu dans des conditions de félicité institutionnelle (Goffman), c'est-à-dire : attendues.

\section{Le carnet du régisseur}

Michel Izard a décelé, au principe de toute institution, « une trame de repères fixes, monuments et sites, qui sont autant de points d'accumulation de mémoire, d'émission et de transmission de messages " (Izard, 1992, p. 119). Je voudrais suggérer, ici, que cette trame de repères n'est ni immobile, ni immuable. Elle est fixe, mais cette fixité résulte de l'action, ou plus exactement, d'une stabilisation qui est à l'œuvre dans l'action. Il y a, dans toute action, une part d'improvisation qui naît du nécessaire ajustement des intentions d'un compositeur avec le savoir-faire de l'acteur et le contexte, vecteur d'un nouvel apprentissage. Autrement dit: le réseau des collaborations nécessaires à la création d'une œuvre musicale est "central en tout point » (Latour). C'est ce que nous montre le témoignage de Ernst Neisel, le régisseur de l'Ensemble Modern.

Le jeudi 17 septembre, au moment de quitter Francfort, Ernst Neisel est confiant: "Tout va bien se passer. C'est juste une question de coordination entre New York, Francfort et Munich. Nous avons déjà monté ce programme l'an dernier, alors ça tourne. Demain, vendredi, ça va être une journée très éprouvante. On ne va pas dormir de la nuit et on va tout installer, à Munich. Mais je ne suis pas inquiet, nous avons l'habitude de travailler ensemble. La preuve : cette fois, je n'ai pas eu besoin de papier. D'habitude, j'ai toujours des papiers que je gribouille. Et là, je note tout au crayon, je fais des schémas que personne ne comprend, mais c'est juste pour moi, pour que je me souvienne des choses. Mais ce programme, on l'a déjà fait plusieurs fois, alors je n'ai pas besoin de petits papiers. " Ensemble, nous essayons de compter le nombre de techniciens : au minimum 22 personnes sont mobilisées, plus le concierge, qui passe deux nuits blanches. Soit : 8 techniciens du Muffathalle, 4 chauffeurs ( 2 camions de 7,5 tonnes), 2 stage managers (Ernst et Michael), 1 ingénieur du son et son assistant, le projectionniste de Jack Young, Steve Ehrenberg pour l'éclairage et son technicien, Deter Reeps qui s'occupe de la scène, plus le camion qui apporte le matériel électrique depuis Nuremberg. 
30 « Il y a une part d'improvisation, dans ce métier, c'est ça qui me plaît, me dit Ernst. Brusquement, quelque chose ne va pas. Le pied d'un marimba casse, par exemple. Eh bien je dois imaginer une solution tout de suite. Ou encore, ça m'est arrivé l'autre jour, à Turin, une société locale installe l'électricité. C'est la première fois qu'elle faisait l'installation dans cette église. Et tout d'un coup, juste avant le concert, tout saute. La panique. Eh bien, moi, dès qu'on était arrivé sur les lieux, j'avais repéré la façon dont l'électricité avait été montée. J'ai réparé tout de suite. C'est comme une anticipation. À Paris, quand on a joué Hindenburg, un piano ne marchait pas. Le branchement ne fonctionnait pas : le sampler était raccordé aux deux pianos. Eh bien, on s'est arrangé. J'ai proposé qu'un pianiste joue les deux parties. C'était la solution effectivement, et personne ne s'est rendu compte de rien.

31 La première fois que je suis arrivé ici, je me suis dit : "Oh là là... Comment je vais faire pour me souvenir de tout ça ?" Alors j'ai imaginé que ça pouvait fonctionner de deux manières : $1^{\circ}$ ) la liste $; 2^{\circ}$ ) le repérage sur les lieux. Maintenant, tu vois, je n'ai presque plus besoin de liste. Je me dis : “Voyons, qui joue ? De quoi a-t-il besoin ?" Alors, comme ça fait un an et demi que je suis ici,j'arrive maintenant à me souvenir exactement de ce qu'il faut. C'est une question de mémoire, et d'initiative.

Alors voilà, par exemple, ce qui s'est passé avant qu'on aille à Paris pour le Festival d'Automne. Roman Paska, le metteur en scène, était très nerveux. Il ne voulait pas que les déplacements des musiciens et des chanteurs sur scène s'entendent pendant l'opéra. Il était obsédé par cette idée. Il voulait trouver la solution. Moi, j'en avais une : il fallait du caoutchouc pour le sol de Three Tales. J'avais pensé à ça, mais lui n'était pas vraiment d'accord. Alors, il a fallu négocier. Mais ce n'était pas évident : lui, à New York, et moi, ici, à Francfort. Ça n'a vraiment pas été facile. À la fin, il était d'accord, mais il a fallu batailler longtemps. Tu comprends, ce n'est pas facile: tu négocies quelque chose de concret (une matière, une installation, un coût) mais, en même temps, rien n'est concret dans la négociation, puisque tout est dans les esprits... et dans le fax.

En fait, dans un concert, il y a deux concerts : celui que les gens écoutent (ça, c'est la musique) et celui qui est fait de l'agencement de mille objets, de mille choses, de milles actions coordonnées et qui doivent se dérouler au bon moment pour que le concert ait lieu. Moi, je suis dans ce niveau là. Je considère mon travail comme une écriture, comme une composition, que je fais moi-même en fonction des éléments qu'on me donne, et de ce que je sais. »

C'est aussi une autre partition, une troisième, qui se joue là. Ernst Neisel a parfaitement mentalisé l'organisation endogène d'une activité qui semble organisée autour de deux axes : mémoire et disponibilité. Ernst veut être attentif aux objets, aux relations qui l'entourent. Il les capte pour fabriquer l'œuvre, c'est-à-dire sa propre "partition ». Ernst Neisel n'a jamais d'avis sur le résultat sonore du concert, sur l'interprétation des musiciens. Pour lui, un concert réussi est un concert dans lequel tout a fonctionné d'une manière optimale et, si possible, avec quelques ratés qu'il aura su, lui, arranger. Ernst Neisel trace des plans, des schémas qu'il fourre dans ses poches. Il me dit qu'il n'en a plus, mais ses poches en sont remplies. Il n'a jamais voulu me les montrer. C'est son intimité, ce sont ces "trucs à lui ", c'est sa "partition intérieure ». Ces petits papiers lui permettent de fixer des états d'avancement de sa création, de fixer des états d'avancement qui, pour le dire ici avec les mots de James J.Gibson, sont autant

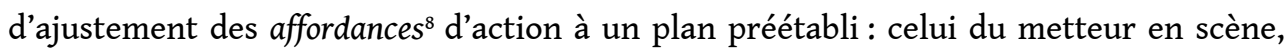


qui pense, peut-être, que Ernst Neisel est le simple exécutant de son plan d'action labellisé9.

Or voilà, si je change d'échelle et que je cherche à séquentialiser le cours d'action, je découpe des unités qui me paraissent significatives dans les comportements (par exemple, ces anecdotes qui font de Ernst Neisel un héros). En portant attention aux modalités de la présence d'Ernst Neisel dans l'espace des interactions, je me situe au plan de "l'action située » (Suchman, 1987), c'est-à-dire non plus au plan de «la préparation mentale de l'action mais son effectuation concrète en contexte " (Quéré, 1997, p. 169). Or, une analyse de l'action située pourrait-elle évacuer ce qui est de l'ordre des représentations collectives pour comprendre « ce » qui se passe quand Ernst Neisel s'engage dans une action réglée?

\section{Le second concert}

J'essaye autrement. Peut-on envisager toute action située comme le point d'aboutissement d'une chaîne causale mobilisant des procédures inscrites dans un continuum temporel et procédant d'une intention liminaire pour aboutir à l'exécution de la tâche, en passant par une étape de planification? Ce découpage idéal en plan d'action, d'une part, et action située, d'autre part, puis la segmentation des dispositifs de planification en choix locaux - dont une logique des enchaînements garantirait la cohésion de l'ensemble «de telle sorte que les buts globaux que [le dispositif de planification] vise à réaliser soient atteints " (Kirsch, 1990, p. 113) - nous permettrait de saisir la rationalité du dispositif de gestion du projet de Steve Reich en entrant dans l'enceinte mentale de l'acteur. Alors, on pourrait observer que les choix faits par l'acteur en situation sont autant de bifurcations qu'il décide d'emprunter à chaque moment opportun, rationnellement, et d'une manière telle, que l'on pourrait prédire sa conduite du point de vue du plan. Pour le dire d'une façon lapidaire, le plan servirait à contrôler l'action. La rationalité du projet pourrait alors se mesurer à l'aune de la rationalité de l'exécution de la tâche. Dans ce secteur de la recherche en sociologie cognitive, la réalisation des tartes aux pommes est dotée d'une valeur canonique : « la recette est un programme d'action, transmis et appris, et la confection du plat s'ajuste aux instructions de la recette » (Conein, 1990, p. 101). Mais peut-on réduire les humains à l'état d'agents situés ? Tout se jouerait-il dans la recette, dans la partition ?

Tout ne se joue pas dans la partition qu'écrit le compositeur, tout ne se joue pas non plus dans la partition que lisent les musiciens, tout ne se joue pas non plus dans la partition du responsable de festival, ni dans celle du metteur en scène, ou dans celles que le régisseur fourre dans ses poches. Toute action se déroule sur la base d'une adaptation incessante, qui permet à chacun d'ériger son environnement en une mémoire externe pour y puiser, le moment venu, les ressources de son comportement ou, pour le dire avec les mots de Francis Chateauraynaud, qui permet à chacun "d'improviser dans les règles » (Chateauraynaud, 1997). Après tout, n'est-ce pas à cela que se mesure la compétence de l'acteur, à sa capacité à improviser dans les règles ?

Pour autant, on ne peut se livrer à une conception atomiste de l'œuvre musicale et dire, par exemple, que l'on pourrait remonter à l'œuvre en combinant, dans une chaîne associative, l'ensemble des éléments de représentation. La production d'une œuvre musicale ne se réduit pas à la somme des actions de chacun. Vincent Descombes nous invite à ne pas céder au "mirage des sujets collectifs» (1996). Dans toute action 
collective, il y a " autre chose » qui intervient que la somme des actions individuelles. Cet " autre chose " nous invite à analyser l'action collective, non pas comme une suite d'emboîtements successifs, mais comme une série d'ajustements. Une équipe de rugby se compose de quinze joueurs dont on peut dresser la liste, mais l'action de l'équipe ne se réduit pas à la somme des actions individuelles de ses joueurs. Cela implique que tout acte significatif actualise la présence d'un système de lois et d'usages, mais ne se réduit pas à un pur décalque du système des lois. À l'inverse, ces capacités s'enracinent dans notre corporéité biologique. Dès lors, appréhender l'action implique d'articuler la règle à la présence corporelle de l'agent en situation, d'articuler l'étude de l'expérience humaine sous son aspect corporel et culturel à la cognition humaine ${ }^{10}$.

Ce que revendique Ernst Neisel, tout au long de son témoignage, c'est une part de créativité, c'est-à-dire une place pour le risque. Car, dans l'enchaînement des situations, la responsabilité ne se réduit pas à une responsabilité statutaire. Elle est le résultat d'un processus d'imputation qui naît dans l'interaction. Pour le dire à la manière de Paul Ricœur, «ce sont des phénomènes comme l'initiative, l'intervention, où se laisse surprendre l'immixtion de l'agent de l'action dans le cours du monde, immixtion qui cause effectivement des changements dans le monde » (Ricœur, 1995, p. 56). La responsabilité est à comprendre, ici, comme une responsabilité en acte. Ernst Neisel entend maîtriser les situations dans lesquelles il est impliqué. Cette maitrise repose sur trois instances : mémoire, compétence, disponibilité.

1. La mémoire passe ici par une mnémotechnie. C'est une manière de réduire l'incertitude de chacune des actions dans lesquelles il se trouve impliqué. En consignant sur un morceau de papier, qu'il ne veut montrer à personne, les éléments pertinents des actions qu'il aura à accomplir, Ernst Neisel opère une réduction de la situation. Jack Goody parlerait d'une domestication de la pensée, Claude Lévi-Strauss d'une réduction pour qualifier ce passage de stimuli divers vers une représentation visuelle : « la vertu intrinsèque du modèle réduit est qu'il compense la renonciation à des dimensions sensibles par l'acquisition de dimensions intelligibles » (Lévi-Strauss, 1962, p. 39). Quels sont les enjeux de cette cartographie?

D'une part, la transposition graphique autorise le retour en arrière et la comparaison, donc la maitrise (Goody, 1979). L'écriture est une manière de mettre entre parenthèses la dimension temporelle des actions à accomplir. D'autre part, le souci d'un repérage spatial est une constante de l'activité humaine. C'est en stabilisant son environnement que l'être humain réduit l'incertitude et rend le monde familier: "Le monde fonctionne comme extension physique de notre système de représentation. [Il] sert de rappel de son propre état, et sa structure physique sert d'outil » (Norman, 1993, p. 18). Ce déplacement vers un espace visuel permet à l'agent de se libérer de la pression du temps et d'apprécier, en un clin d'œil, la situation dans son ensemble. En même temps, le support papier permet à Ernst Neisel de dominer la situation comme le cartographe domine le monde. Bruno Latour a montré que la cartographie est de ces «moyens grâce auxquels la mobilité, la stabilité ou la capacité de combinaison assurent l'action à distance » (Latour, 1995, p. 535). La cartographie est un moyen d'annexer l'inconnu, de le ramener au familier et de le maîtriser. L'opération d'inscription de repères mnémotechniques permet à Ernst Neisel de dominer la situation, c'est-à-dire de la maîtriser en la composant.

2. La compétence intervient, ici, en tant que compétence technique mais aussi en tant qu'assignation statutaire socialement définie. C'est parce qu'on lui reconnaît une 
compétence certaine, qu'Ernst Neisel peut se sentir autorisé à improviser, ou, pour le dire moins crûment, à prendre des initiatives.

3. La disponibilité, enfin, tient à cette capacité de se maintenir en éveil, de rester vigilant. À ce stade de l'analyse, il nous faut intégrer le niveau des pratiques corporelles qui s'insèrent dans les réseaux de conventions et d'objets techniques. Bref : intégrer la question des modalités pratiques et les aspects argumentatifs ou normatifs de l'action. Cela n'est jamais aussi clair qu'au moment où Ernst Neisel évoque la négociation par fax.

Le rapport homme/matière est réglé dans une cascade d'unités intégrées où la place primordiale revient au travail perceptuel. Toute action d'Ernst prend appui sur des régularités: une forme de routine qui lui permet d'être disponible pour guetter le surgissement de l'inattendu ${ }^{11}$. Ces régularités s'organisent autour de points fixes fournis par l'environnement, qu'il contribue ainsi à renforcer. Du coup, son action se traduit par une forme de stabilisation pour les autres acteurs et ouvre sur de nouvelles expériences. Cette «stabilité de l'institution» dont parlait Michel Izard n'est pas une immobilité. C'est une stabilité en mouvement, qui naît des rapports mutuels qui naissent dans l'action, qui naissent d'une multiplicité d'interactions structurantes. Ramener l'inconnu au connu, l'étranger au familier, la nouveauté à la routine, telle est sa fonction. De la multiplication de l'accomplissement de ces tâches naît la possibilité d'une action coordonnée. Quand Ernst Neisel prépare l'espace scénique, il installe les musiciens dans un cadre qui leur est familier, de sorte que leur disponibilité pour l'exécution soit totale. Ils savent qu'ils auront un pupitre pour poser leur partition, un projecteur pour les éclairer, qu'ils auront les mêmes voisins de pupitre qu'à la répétition, que le chef sera au même endroit et qu'il se passera telle ou telle chose dans le déroulement du concert. Ernst Neisel se charge de faire en sorte que le référentiel qu'ont connu les musiciens pendant les répétitions, à Francfort, se retrouve, inchangé, au moment du concert à Munich.

Cet appel incessant aux facultés perceptuelles nous amène à récuser l'idée d'une hypothèse mentaliste qui considérerait que l'agent mémorise et anticipe parce qu'il a incorporé l'ensemble des scénarios les plus probables. La prolifération des interactions structurantes par lesquelles on aboutit, finalement, à la fabrication de musique, naît dans le principe d'enaction, que Varela définit en deux points: "(1) la perception consiste en une action guidée par la perception ; (2) les structures cognitives émergent des schèmes sensori-moteurs récurrents qui permettent à l'action d'être guidée par la perception » (Varela, 1993, p. 234-235). En interrogeant la façon dont le sujet percevant parvient à guider ses actions dans une situation locale, l'enaction fait de la structure sensori-motrice le point de référence de l'action: l'enaction est bien une cognition incarnée. Or, au terme de notre inventaire des situations et de l'intrication des rapports au sein des réseaux, ne devrait-on pas renverser la perspective et " partir d'un système de contrainte ou de représentation, lui-même objectivé dans des plans, des procédures, des instruments, des collectifs, et les mettre à l'épreuve de la variation, sonder les ressources de l'environnement pour engendrer des prises dérivées " (Chateauraynaud, 1997, p. 125) ? L'exécution de la tâche ne s'apparente plus alors à un simple décalque du plan d'action. Pour chacun des acteurs qui participent de cette longue chaîne chaotique qui fait l'œuvre musicale, qui la fabrique en rendant possible sa présentification au concert, l'exécution de la tâche s'apparente plutôt à un engagement dans une "improvisation régulatrice», c'est-à-dire à une prise de responsabilité dans une 
prolifération de situations dans lesquelles, agissant au nom de l'institution musicale, il régule le flux des échanges et organise ainsi les conditions d'effectuation du projet de ce compositeur qui, un jour, dans un chalet en rondins de bois, quelque part dans la région de Rochester, dans le Vermont, inscrivit des notes, à l'encre noire, sur du papier à musique.

\section{BIBLIOGRAPHIE}

Born Georgina, Rationalizing Culture: IRCAM, Pierre Boulez and the Institutionalization of the Musical Avant-Garde, Berkeley/Los Angeles/London, University of California Press, 1994.

Chateauraynaud Francis, « Vigilance et transformation. Présence corporelle et responsabilité dans la conduite des dispositifs techniques ", Réseaux, n 85, 1997, p. 101-127.

Cheyronnaud Jacques, «Ethnologie et musique. L'objet en question », Ethnologie française, $\mathrm{n}^{\circ} 3$, 1997, p. 382-393.

Cohen Yves, « Le xxe siècle commence en 1900 ", Alliage, nº 21, 1994, p. 88-104.

Conein Bernard, « Cognition située et coordination de l'action : la cuisine dans tous ses états », Réseaux, $\mathrm{n}^{\circ}$ 43, 1990, p. 99-110.

Descombes Vincent, Les Institutions du sens, Paris, Minuit, 1996.

Dewey John, Art as experience [ $1^{\text {st }}$ ed. 1934], New York, Peregee book, 1980.

Genette Gérard, L'CEuvre de l'art. Immanence et transcendance, Paris, Seuil, 1994.

Genette Gérard, L'CEuvre de l'art. La relation esthétique, Paris, Seuil, 1997.

Gibson James J., The Ecological Approach to Visual Perception, Hillsdale/New Jersey, Lawrence Erlbaum Associates, 1979.

Goffman Erving, La mise en scène de la vie quotidienne. Les territoires du moi, Paris, Minuit, 1973.

Goodman Nelson, Langages de l'art, Nîmes, Jacqueline Chambon (trad. de l'éd. am. de 1968), 1990.

Goody Jack, La Raison graphique, Paris, Minuit, 1979.

Hennion Antoine, La Passion musicale. Une sociologie de la médiation, Paris, éditions Métailié, 1993.

Izard Michel, L'Odyssée du pouvoir, un royaume africain : État, société, destin individuel, Paris, éditions de l'EHESS, 1992.

Kirsch David, « Préparation et improvisation », Réseaux, n 43, 1990, p. 11-120.

Laborde Denis, De Jean-Sébastien Bach à Glenn Gould. Magie des sons et spectacle de la passion, Paris, L'Harmattan, 1996.

Laborde Denis, Musiques à l'école, Paris, éditions Bertrand-Lacoste, 1998.

Latour Bruno, « Le topofil de Boa-Vista », Raisons pratiques, nº 4, 1993, p. 187-216.

Latour Bruno, La Science en action. Introduction à la sociologie des sciences, Paris, Gallimard (Folio essais), 1995. 
Levinson Jerold, L'Art, la musique et l'histoire, Paris, éditions de l'Éclat (trad. de l'éd. am. de 1990), 1998.

Levi-Strauss Claude, La Pensée sauvage, Paris, librairie Plon, 1962.

Menger Pierre-Michel, « Machines et novateurs », Raisons pratiques, nº 4, 1993, p. 165-186.

Menger Pierre-Michel, Les Laboratoires de la création musicale. Acteurs, organisations et politique de la recherche musicale, Paris, La Documentation française, 1994.

Norman Donald A., « Les Artefacts cognitifs », Raisons pratiques, n 4, 1993, p. 15-34.

Piette Albert, Ethnographie de l'action. L'observation des détails, Paris, éditions Métailié, 1996.

Putnam Hilary, Représentation et réalité, Paris, Gallimard (trad. de l'éd. am. de 1988), 1990.

Quere Louis, « La situation toujours négligée ?», Réseaux, nº 85, 1997, p. 163-192.

Revel Jacques, (dir.), Jeux d'échelles. La micro-analyse à l'expérience, Paris, Seuil/Gallimard, 1996.

Ricœur Paul, Le Juste, Paris, Esprit, 1995.

Rosen Charles, Aux confins du sens. Propos sur la musique, Paris, Seuil (La librairie du Xx siècle), (trad. de l'éd. am. de 1994), 1998.

Schaeffer Jean-Marie, Les Célibataires de l'art. Pour une esthétique sans mythes, Paris, Gallimard (nrf essais), 1996.

Strauss Anselm, «L'influence réciproque de la routine et de la non-routine dans l'action », in Pierre-Michel Menger et Jean-Claude Passeron (dir.), L'Art de la recherche. Essais en l'honneur de Raymonde Moulin, Paris, La Documentation française, 1994, p. 351-366.

Suchman Lucy A., Plans and Situated Actions. The Problem of Human-Machine communication, Cambridge, CUP, 1987.

Varela Francisco, Thompson Evan, Rosch Eleanor, L'Inscription corporelle de l'esprit. Sciences cognitives et expérience humaine, Paris, Seuil, (La couleur des idées), 1993.

\section{NOTES}

1. Suivant un partage définitoire devenu classique en histoire de l'art, je distingue l'artistique de l'esthétique sur la base du critère d'intentionnalité. L'esthétique est, communément, le lieu d'une mobilisation émotionnelle, qu'elle soit ou non intentionnelle. L'artistique s'applique à toute production d'artefacts intentionnellement esthétique (indépendamment de la "valeur» attribuée à l'artefact). L'introduction que Gérard Genette a rédigée en ouverture du tome 1 de L'CEuvre de l'art (1994, p. 7-32) s'offre comme une remarquable synthèse des efforts engagés pour clarifier la terminologie en usage.

2. Si, à la suite de Jacques Revel, nous pouvons remarquer que « la démarche micro-historique est devenue, ces dernières années, l'un des lieux importants du débat épistémologique entre les historiens » (Revel, 1996, p. 15), il est sans doute possible de faire un même constat pour la sociologie. Mais il est remarquable que les apports de l'interactionnisme, par exemple, n'aient fait qu'une entrée fort récente dans l'ethnologie européenne. Cf. le Forum on Anthropology in/of France (infra bibliographie Marcus, 1999).

3. Ce qui implique de considérer que le domaine des tâches envisageables peut être spécifié ad infinitum. Nous ne sommes pas ici dans un domaine de tâches clairement circonscrit comme l'est, par exemple, l'espace du jeu d'échec qui est devenu le parangon de bien des études sur la cognition située. Là, en effet, il est relativement aisé de repérer les différents constituants de cet 
espace, d'isoler des éléments discrets dans la réalisation du jeu (échiquier, pions, règles, dispositions, attention, stratégies...) Dans le domaine de la création musicale, les efforts de planification se heurtent à des actions en situation qui ne sont pas nécessairement formalisables en ces termes. Le monde de la création musicale ne se termine pas en un certain point. Il consiste en l'agencement de niveaux de détails reculant à l'infini, où l'expérience accumulée par les agents dans des situations concrètes antérieures s'ajuste à la planification élaborée pour ce concert précis. L'ensemble de cette recherche vise à tenter une reformulation des oppositions canoniques dans les sciences sociales entre le collectif et l'action individuelle, entre l'espace public et l'espace privé, en distinguant la façon dont plusieurs régimes d'inscription dans l'espace social de l'interaction coexistent.

4. Les guillemets sont durcis dans mon propos. Chaque fois qu'il sera question de "Steve Reich", je considérerai le compositeur et sa réputation, c'est-à-dire ses œuvres passées et le compositeur comme figure de l'admiration, avec le rendement social attaché à la mise en circulation de son nom.

5. Une négociation ultérieure renversera finalement l'ordre des concerts. Three Tales sera donné à Paris le 13 septembre 2001, à Berlin le 29 septembre. Mais rien n'est encore stabilisé. Des changements sont susceptibles d'intervenir tant que les programmes ne sont pas imprimés et les concerts annoncés.

6. Parler de "partition » à propos de la planification composée par l'administrateur implique de mettre en circulation une conception élargie du mot. À la suite de Nelson Goodman, je considère que le mot partition désigne "toute espèce de caractères décrits dans un système notationnel et non pas simplement dans la notation musicale» (Goodman, 1990, p. 218). Sans doute comprendon mieux ce sens élargi si l'on fait référence au terme anglais score, qui renvoie à une fonction inscriptive en même temps qu'il désigne, de façon plus technique, toute marque qui sert de repère. Cf. Goodman (1990, p. 217 sq.), ainsi que les précisions apportées par son traducteur, Jacques Morizot, à la page 261 des Langages de l'Art.

7. C'est, par exemple, tout le travail que Bruno Monsaingeon réalise avec une maîtrise remarquable lorsqu'il filme Glenn Gould. Dans ces célèbres films sur le pianiste canadien, le réalisateur doit faire en sorte que chaque téléspectateur se sente concerné, adhère au discours de l'opinion affichée par Glenn Gould, il doit susciter l'engagement, coordonner les implications émotionnelles, promouvoir Glenn Gould jouant Bach en spectacle de l'émotion. Mais il faut, pour cela, que l'écran devienne invisible, que l'infrastructure technologique disparaisse pour cultiver l'illusion d'une présence de "Glenn Gould». Je me permets de renvoyer aux pages de mon ouvrage sur ce thème (infra bibliographie, 1997, p. 63-102).

8. C'est au huitième chapitre de son ouvrage, The Ecological Approach to Visual Perception (1979), que James J. Gibson explicite sa théorie des affordances. Il fonde le néologisme sur le verbe to afford, survenir. Transposé tel quel en français, le terme affordances réfère aux informations qui proviennent de l'environnement quand un agent (aussi bien un animal) engage une action. Il « implique la complémentarité de l'animal et de l'environnement »(Gibson, 1979, p. 127).

9. La scénographie est soumise au droit d'auteur. C'est la raison pour laquelle Ernst Neisel n'a pu me remettre une copie du programme scénographique sans l'autorisation du metteur en scène.

10. Ce parti pris implique que notre enquête sur la création des Three Tales ne se comprend pas du même point de vue que celle qu'a menée Pierre-Michel Menger, par exemple, sur les laboratoires de la création musicale (Menger, 1993). Quand P.-M. Menger objective « le réseau des liens tissés entre ces établissements et entre leurs responsables comme l'épure organigrammatique de la centralisation institutionnelle du système musical »(Menger, 1989, p. 20), j'engage une observation participante qui me fait privilégier une démarche noninstitutionnaliste pour saisir la façon dont une institution devient le lieu d'une assignation sémantique. Toutefois, ces deux démarches ne sont pas exclusives l'une de l'autre, comme le 
montre le remarquable travail monographique réalisé par Georgina Born sur l'IRCAM (Born, 1994).

11. Ce qui implique de considérer la routine, non pas comme la simple répétition mécanique de gestes mille fois répétés, mais comme une solution mise au point pour résoudre des configurations problématiques dans lesquelles l'agent se trouve régulièrement impliqué. Ces schémas de comportement standardisé sont susceptibles d'être, à tout moment, érigés en ressource pour l'action: «toute action nouvelle comporte nécessairement des aspects routinisés »(Strauss, 1994, p. 353).

\section{AUTEUR}

\section{DENIS LABORDE}

Directeur de recherche, CNRS, Centre Marc Bloch, Berlin 